EPJ Web of Conferences 97,00006 (2015)

DOI: 10.1051/epjconf/ 20159700006

(C) Owned by the authors, published by EDP Sciences, 2015

\title{
Resonance dynamics in the PHSD approach
}

\author{
Elena Bratkovskaya ${ }^{1,2, a}$ \\ ${ }^{1}$ Institute for Theoretical Physics, University of Frankfurt, Frankfurt, Germany \\ ${ }^{2}$ Frankfurt Institute for Advanced Studies, 60438 Frankfurt am Main, Germany
}

\begin{abstract}
We present an overview on the resonance dynamics within the microscopic parton-hadron-string dynamics (PHSD) approach which incorporates explicit partonic degrees-of-freedom in terms of strongly interacting quasiparticles (quarks and gluons) in line with an equation-of-state from lattice QCD as well as the dynamical hadronization and hadronic collision dynamics in the final reaction phase. We discuss how the vector meson resonances can be used as a probe of the in-medium effects and demostrate that the low mass dilepton spectra show visible in-medium effects from dynamical vector-meson spectral functions from SIS to SPS energies whereas at RHIC and LHC energies such medium effects become more moderate. We show also that the intermediate mass spectra are dominated by the radiation from the partonic degrees of freedom at RHIC and LHC energies.
\end{abstract}

\section{Introduction}

The resonance production in heavy-ion collisions provides an additional information on the microscopic dynamical processes [1]. The resonances can be produced throughout the whole history of the reaction by hadronization of the Quark-Gluon Plasma (QGP) as well as in the hadronic phase by rescattring. Thus they can provide information about the partonic phase as well as about in-medium effects related to hadronic interactions. The resonances are strongly interacting particles with broad spectral functions, so they have a large width and small life time. Shortly after production the resonances decay into hadrons. Some of them, e.g. vector mesons can decay electromagnetically to di-lepton pairs (correlated electron-positron or $\mu^{+} \mu^{-}$pairs). The hadronic decay mode is dominant whereas the leptonic decay is a very rare process due to the small branching ratio. However, there is a big disadvantage to use the hadronic mode in order to explore in-medium properties of the hadrons: it is very difficult experimentally to identify the products of hadronic decays due to their strong interactions with the environment. In this respect the leptonic mode is more promising since dileptons interact only electromagnetically, i.e. very weakly with the strongly interacting partonic or hadronic medium created in the collisions. Thus dileptons can provide an undistorted information about the different phases of the reactions as well as about in-medium properties of resonances.

\footnotetext{
a e-mail: Elena.Bratkovskaya@th.physik.uni-frankfurt.de
} 


\section{The PHSD approach}

The dynamics of partons, hadrons and strings in relativistic nucleus-nucleus collisions is analyzed here within the Parton-Hadron-String Dynamics approach [2]. In this transport approach the partonic dynamics is based on Kadanoff-Baym equations for Green functions with self-energies from the Dynamical QuasiParticle Model (DQPM) [3] which describes QCD properties in terms of 'resummed' single-particle Green functions. In Ref. [4], the actual three DQPM parameters for the temperaturedependent effective coupling were fitted to the recent lattice QCD results of Ref. [5]. The latter lead to a critical temperature $T_{c} \approx 160 \mathrm{MeV}$ which corresponds to a critical energy density of $\epsilon_{c} \approx 0.5$ $\mathrm{GeV} / \mathrm{fm}^{3}$. In PHSD the parton spectral functions $\rho_{j}(j=q, \bar{q}, g)$ are no longer $\delta$ - functions in the invariant mass squared as in conventional cascade or transport models but depend on the parton mass and width parameters which were fixed by fitting the lattice QCD results from Ref. [5]. We recall that the DQPM allows one to extract a potential energy density $V_{p}$ from the space-like part of the energymomentum tensor as a function of the scalar parton density $\rho_{s}$. Derivatives of $V_{p}$ w.r.t. $\rho_{s}$ then define a scalar mean-field potential $U_{s}\left(\rho_{s}\right)$ which enters into the equation of motion for the dynamical partonic quasiparticles. Furthermore, a two-body interaction strength can be extracted from the DQPM as well from the quasiparticle width in line with Ref. [6]. The transition from partonic to hadronic d.o.f. (and vice versa) is described by covariant transition rates for the fusion of quark-antiquark pairs or three quarks (antiquarks), respectively, obeying flavor current-conservation, color neutrality as well as energy-momentum conservation $[2,4]$. Since the dynamical quarks and antiquarks become very massive close to the phase transition, the formed resonant 'prehadronic' color-dipole states ( $q \bar{q}$ or $q q q$ ) are of high invariant mass, too, and sequentially decay to the groundstate meson and baryon octets increasing the total entropy.

On the hadronic side PHSD includes explicitly the baryon octet and decouplet, the $0^{-}$- and $1^{-}$meson nonets as well, as selected higher resonances as in the Hadron-String-Dynamics (HSD) approach $[7,8]$. Hadrons of higher masses $(>1.5 \mathrm{GeV}$ in case of baryons and $>1.3 \mathrm{GeV}$ for mesons) are treated as 'strings' (color-dipoles) that decay to the known (low-mass) hadrons, according to the JETSET algorithm [9]. Note that PHSD and HSD merge at low energy density, in particular below the critical energy density $\epsilon_{c} \approx 0.5 \mathrm{GeV} / \mathrm{fm}^{3}$. For more detailed descriptions of PHSD and its ingredients we refer the reader to Refs. [3, $4,10,11]$.

\subsection{Production sources}

Dileptons $\left(e^{+} e^{-}\right.$or $\mu^{+} \mu^{-}$pairs) can be emitted from all stages of the reactions as well as a photons. One of the advantages of dileptons compared to photons is an additional 'degree of freedom' - the invariant mass $M$ which allows to disentangle various sources. The following production sources of dileptons in $p+p, p+A$ and $A+A$ collisions are leading [12]

1) Hadronic sources:

- at low invariant masses $(M<1 \mathrm{GeV} c)$ - the Dalitz decays of mesons and baryons $\left(\pi^{0}, \eta, \Delta, \ldots\right)$ and the direct decay of vector mesons $(\rho, \omega, \phi)$ as well as hadronic bremsstrahlung;

- at intermediate masses $(1<M<3 \mathrm{GeV} c)$ - leptons from correlated $D+\bar{D}$ pairs, radiation from multi-meson reactions $\left(\pi+\pi, \pi+\rho, \pi+\omega, \rho+\rho, \pi+a_{1}, \ldots\right)$ - so called ' $4 \pi^{\prime}$ contributions;

- at high invariant masses $(M>3 \mathrm{GeV} c)$ - the direct decay of vector mesons $\left(J / \Psi, \Psi^{\prime}\right)$ and initial 'hard' Drell-Yan annihilation to dileptons $\left(q+\bar{q} \rightarrow l^{+}+l^{-}\right.$, where $\left.l=e, \mu\right)$.

2) 'thermal' QGP dileptons radiated from the partonic interactions in heavy-ion $(A+A)$ collisions that contribute dominantly to the intermediate masses. The leading processes are the 'thermal' $q \bar{q}$ annihilation $\left(q+\bar{q} \rightarrow l^{+}+l^{-}, \quad q+\bar{q} \rightarrow g+l^{+}+l^{-}\right)$and Compton scattering $\left(q(\bar{q})+g \rightarrow q(\bar{q})+l^{+}+l^{-}\right)$. 


\subsection{The PHSD results from SIS to LHC energies}

At energies around $1 \mathrm{AGeV}$ dileptons have been measured in heavy-ion collisions at the BEVALAC in Berkeley by the DLS Collaboration by more than two decades ago. These data led to the so called 'DLS puzzle' because the DLS dilepton yield in $\mathrm{C}+\mathrm{C}$ and $\mathrm{Ca}+\mathrm{Ca}$ collisions at $1 \mathrm{AGeV}$ in the invariant mass range from 0.2 to $0.5 \mathrm{GeV}$ was about five times higher than the results from different transport models at that time using the 'conventional' dilepton sources such as bremsstrahlung, $\pi^{0}, \eta, \omega$ and $\Delta$ Dalitz decays and direct decay of vector mesons $(\rho, \omega, \phi)$ [13]. To solve this puzzle was one of the main motivations to build the HADES (High Acceptance Dilepton Spectrometer) detector at GSI [14]. Indeed the HADES Collaboration could confirm the DLS measurements at $1 \mathrm{AGeV}$ when passing their events for $\mathrm{C}+\mathrm{C}$ through the DLS filter [15]. From the theory side it was argued that the pn bremsstrahlung channel should be sizeably enhanced as compared to the early soft photon calculations [13]. Indeed, a good reproduction of various spectra a different energies could be achieved within the HSD calculations in Ref. [16]. Note, however, that even the bremsstrahlung from pn reactions at these low energies is discussed controversally in the community and not available experimentally. We here report on the actual status of the transport calculations in comparison to the HADES data [17].
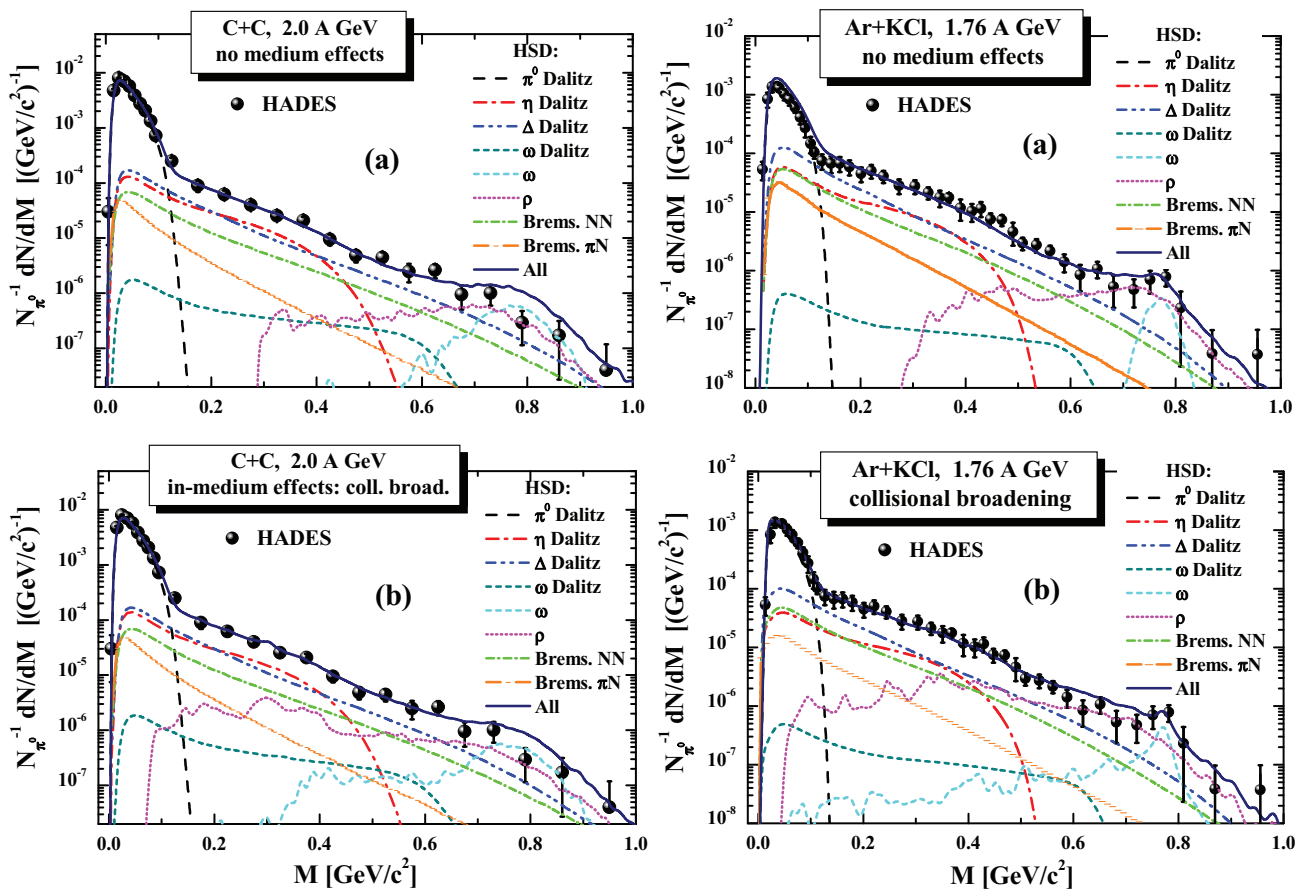

Figure 1. The mass differential dilepton spectra - normalized to the $\pi^{0}$ multiplicity - from HSD calculations for $\mathrm{C}+\mathrm{C}$ at $2 \mathrm{AGeV}$ (1.h.s.) and $\mathrm{Ar}+\mathrm{KCl}$ at $1.76 \mathrm{AGeV}$ (r.h.s.) in comparison to the HADES data [14, 18]. The upper parts (a) shows the case of 'free' vector-meson spectral functions while the lower parts (b) give the result for the 'collisional broadening' scenario. The different colour lines display individual channels in the transport calculation (see legend). The theoretical calculations passed through the corresponding HADES acceptance filter and mass/momentum resolutions.

Fig. 1 (1.h.s.) shows the mass differential dilepton spectra - normalized to the $\pi^{0}$ multiplicity from HSD calculations for $\mathrm{C}+\mathrm{C}$ at $2 \mathrm{AGeV}$ in comparison to the HADES data [18]. The theoretical 
calculations passed through the corresponding HADES acceptance filters and mass/momentum resolutions which leads to a smearing of the spectra at high invariant mass and particularly in the $\omega$ peak region. The upper part shows the case of 'free' vector-meson spectral functions while the lower part presents the result for the 'collisional $\rho$ broadening' scenario. Here the difference between in-medium scenarios is of minor importance and partly due to the limited mass resolution which smears out the spectra. Fig. 1 (r.h.s.) displays the mass differential dilepton spectra - normalized to the $\pi^{0}$ multiplicity - from HSD calculations for the heavier system $\mathrm{Ar}+\mathrm{KCl}$ at $1.76 \mathrm{AGeV}$ in comparison to the HADES data [14]. The upper part shows again the case of 'free' vector-meson spectral functions while the lower part gives the result for the 'collisional broadening' scenario. Also in this data set the enhancement around the $\rho$ mass is clearly visible. For the heavier system the 'collisional broadening' scenario shows a slightly better agreement with experiment than the 'free' result and we expect that for larger systems the difference between the two approaches increases. The effects from a broadened vectormeson spectral function is more visible for $\mathrm{Au}+\mathrm{Au}$ collisions. The respective data have been taken by the HADES Collaboration and are currently analyzed. For detailed predictions we refer the reader to Ref. [17].

We note that with increasing mass $\mathrm{A}+\mathrm{A}$ of the system the low mass dilepton regime from roughly 0.15 to $0.5 \mathrm{GeV}$ increases due to multiple $\Delta$-resonance production and Dalitz decay. The dileptons from intermediate $\Delta$ 's, which are part of the reaction cycles $\Delta \rightarrow \pi N ; \pi N \rightarrow \Delta$ and $N N \rightarrow N \Delta ; N \Delta \rightarrow N N$, escape from the system while the decay pions do not [17]. With increasing system size more generations of intermediate $\Delta$ 's are created and the dilepton yield enhanced accordingly. In inclusive $\mathrm{C}+\mathrm{C}$ collisions there is only a moderate enhancement relative to scaled $\mathrm{p}+\mathrm{p}$ and $\mathrm{p}+\mathrm{n}$ collisions due to the small size of the system while in $\mathrm{Ar}+\mathrm{KCl}$ reactions already several (3-4) reactions cycles become visible. Similar finding has been obtained within the IQMD transport model - cf. Figs. 25, 27, 29 in Ref. [17]. This effect enhances with the system size and reaches a factor of 4.5-5 for $\mathrm{Au}+\mathrm{Au}$ minimal bias at $1.25 \mathrm{~A} \mathrm{GeV}$ as illustrated in Fig. 2 which presents the ratio $\left(1 / N_{\pi^{0}}^{A A} d N^{A A} / d M\right) /\left(1 / N_{\pi^{0}}^{N N} d N^{N N} / d M\right)$ of the mass differential dilepton spectra - normalized to the $\pi^{0}$ multiplicities - obtained in HSD calculations. Displayed are the ratios of minimal bias $\mathrm{C}+\mathrm{C}, \mathrm{Ar}+\mathrm{KCl}$, $\mathrm{Au}+\mathrm{Au}$ collisions and of the isospin-averaged reference spectra $N N=(p n+p p) / 2$ at the same energy. The lower plot depicts the same ratios but for the dilepton spectra after $\eta$ Dalitz yield subtraction. Additionally to the $\Delta$ regeneration, the $p N$ bremsstrahlung - which scales with the number of collisions and not with the number of participants, i.e. pions - contribute to the enhancement of the ration in Fig. 2. We note that the preliminary HADES data for central Au+Au collisions at $1.23 \mathrm{~A} \mathrm{GeV}$ show an enhancement factor up to 8-10 [19]. The detailed calculations with the proper experimental acceptance are required for the quantitative conclusions, however, based on our study in Ref. [17] we mainly attribute this enhancement to two origins: i) the bremsstrahlung radiation from $p n$ and $p p$ reactions which does not scale with the pion number (i.e. the number of participants) rather with the number of elementary elastic collisions. ii) the shining of dileptons from the 'intermediate' $\Delta$ 's, which take part in the $\Delta \rightarrow \pi N$ and $\pi N \rightarrow \Delta$ reaction cycle. This cycle produces a number of generations of $\Delta$ 's during the reaction which increases with the size of the system. At the end only one pion is produced but each intermediate $\Delta$ has contributed to the dilepton yield because emitted dileptons do not get absorbed. This leads to an enhancement of the dilepton yield as compared to the final number of pions. Thus, the enhancement confirms the predictions of transport theories that in heavy-ion collisions several generations of $\Delta$ 's are formed which decay and are recreated by $\pi N \rightarrow \Delta$ reactions. Accordingly, the dilepton data from $A A$ reactions shed light on the $\Delta$ dynamics in the medium.

Dileptons from heavy-ion collisions at SPS energies have been measured in the last decades by the CERES [20] and NA60 [21] Collaborations. The high accuracy dimuon NA60 data provide a unique possibility to subtract the hadronic cocktail from the spectra and to distinguish different 

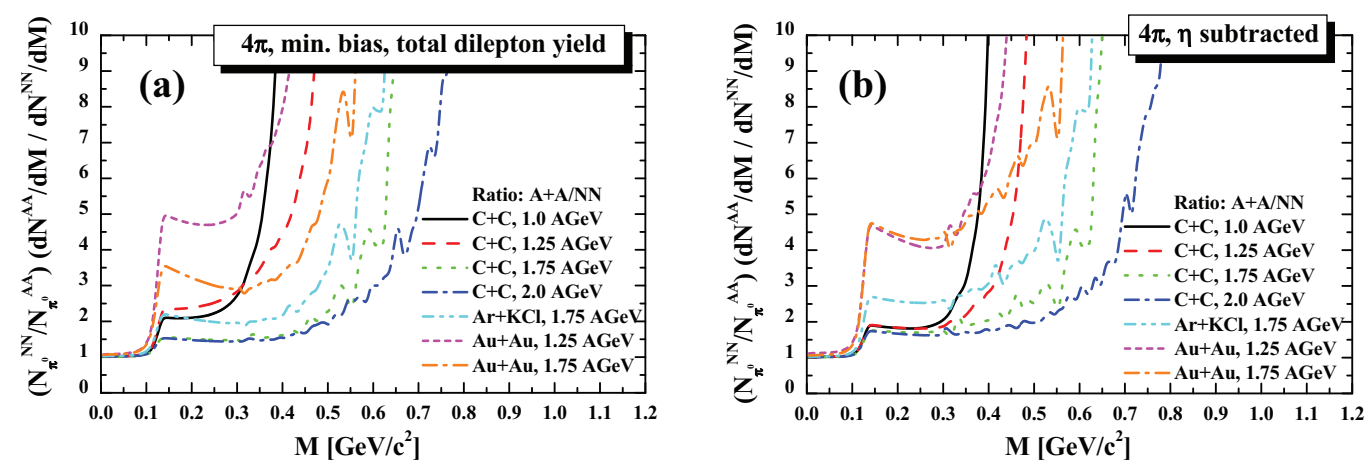

Figure 2. Left plot (a): The ratio $\left(1 / N_{\pi^{0}}^{A A} d N^{A A} / d M\right) /\left(1 / N_{\pi^{0}}^{N N} d N^{N N} / d M\right)$ of the invariant mass differential dilepton $4 \pi$ spectra - normalized to the $\pi^{0}$ multiplicity - from HSD calculations for minimal bias $A+A$ collisions: We display $\mathrm{C}+\mathrm{C}, \mathrm{Ar}+\mathrm{KCl}, \mathrm{Au}+\mathrm{Au}$ collisions in comparison to the isospin-averaged reference spectra $N N=(p n+$ $p p) / 2$ at 1.0, 1.25, 1.75, 2.0 AGeV. Right plot (b): the same ratios but for the dilepton spectra after $\eta$ Dalitz yield subtraction.

in-medium scenarios for the $\rho$-meson spectral function such as a collisional broadening and dropping mass $[22,23]$. The main messages obtained by a comparison of the variety of model calculations (see e.g. $[22,24,25])$ with experimental data can be summarized as

(i) the low mass spectra $[20,21]$ provide a clear evidence for the collisional broadening of the $\rho$-meson spectral function in the hot and dense medium;

(ii) the intermediate mass spectra above $M>1 \mathrm{GeV} / c^{2}$ [21] are dominated by partonic radiation;

(iii) the rise and fall of the inverse slope parameter of the dilepton $p_{T}$-spectra (effective temperature) $T_{\text {eff }}$ [21] provide evidence for the thermal QGP radiation;

(iv) isotropic angular distributions [21] are an indication for a thermal origin of dimuons.

An increase in energy from SPS to RHIC has opened new possibilities to probe by dileptons a possibly different matter at very high temperature, i.e. dominantly in the QGP stage, created in central heavy-ion collisions. The dileptons ( $e^{+} e^{-}$pairs) have been measured first by the PHENIX Collaboration for $p p$ and $A u+A u$ collisions at $\sqrt{s}=200 \mathrm{GeV}$ [26]. A large enhancement of the dilepton yield relative to the scaled $p p$ collisions in the invariant mass regime from 0.15 to $0.6 \mathrm{GeV} / c^{2}$ has been reported for central $\mathrm{Au}+\mathrm{Au}$ reactions. This observation has stimulated a lot of theoretical activity (see the model comparison with the data in Ref. [26]). The main messages - which hold up-to-now - can be condensed such that the theoretical models, which provide a good description of $p p$ dilepton data and peripheral $A u+A u$ data, fail in describing the excess in central collisions even with in-medium scenarios for the vector-meson spectral function [24]. The missing strengths might be attributed to low $p_{T}$ sources [27]. On the other hand the intermediate mass spectra are dominated by the QGP radiation as well as leptons from correlated charm pairs $(D+\bar{D})[24,27,28]$.

In this respect it is very important to have independent measurements which have been carried out by the STAR Collaboration [30]. Fig. 3 shows the comparison of STAR data of midrapidity dilepton yields (1.h.s.) and its ratios (r.h.s.) to the 'cocktail' for 0-10\%, 10-40\%, 40-80\%, 0-80\% central $\mathrm{Au}+\mathrm{Au}$ collisions at $\sqrt{s_{N N}}=200 \mathrm{GeV}$ in comparison to the theoretical model predictions from the PHSD approach and the expanding fireball model of Rapp and collaborators. As seen from Fig. 3 the excess of the dilepton yield over the expected cocktail is larger for very central collisions and consistent with the model predictions including the collisional broadening of the $\rho$-meson spectral 

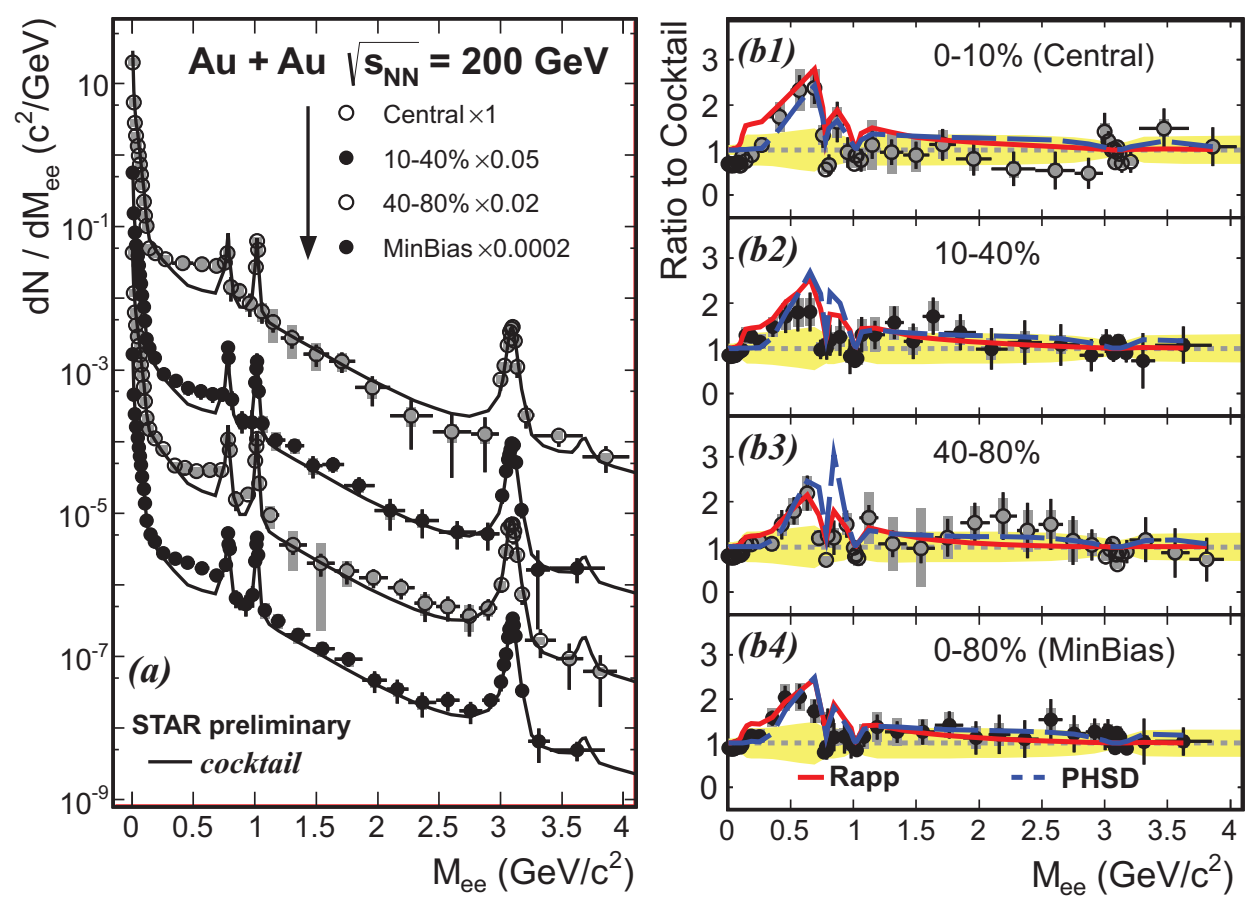

Figure 3. Centrality dependence of the midrapidity dilepton yields (left) and its ratios (right) to the 'cocktail' for $0-10 \%, 10-40 \%, 40-80 \%, 0-80 \%$ central Au+Au collisions at $\sqrt{s}=200 \mathrm{GeV}$ : a comparison of STAR data with theoretical predictions from the PHSD ('PHSD' - dashed lines) and the expanding fireball model ('Rapp' - solid lines). The figure is taken from Ref. [32].

function at low invariant mass and QGP dominated radiations at intermediate masses. Moreover, the recent STAR dilepton data for $\mathrm{Au}+\mathrm{Au}$ collisions from the Beam Energy Scan (BES) program for $\sqrt{s_{N N}}=19.6,27,39$ and $62.4 \mathrm{GeV}[29,31,32]$ are also in line with the expanding fireball model (as well as PHSD) predictions with a $\rho$ collisional broadening [32]. According to the PHSD calculations the excess is increasing with decreasing energy due to a longer $\rho$-propagation in the high baryon density phase (see Fig. 3 in [29]).

The upcoming PHENIX data for central Au+Au collisions - obtained after an upgrade of the detector - together with the BES-II RHIC data should provide finally a consistent picture on the low mass dilepton excess in relativistic heavy-ion collisions. On the other hand, the upcoming ALICE data [33] for heavy-ion dileptons for $\mathrm{Pb}+\mathrm{Pb}$ at $\sqrt{s}=2.76 \mathrm{TeV}$ will give a clean access to the dileptons emitted from the QGP [28, 34]. In Fig. 4 (1.h.s.) we present the PHSD predictions for central $\mathrm{Pb}+\mathrm{Pb}$ collisions [34] in the low mass sector for a realistic lepton $p_{T}$ cut of $1 \mathrm{GeV} / \mathrm{c}$. It is clearly seen that the QGP sources and contribution from correlated $D \bar{D}$ pairs are subleading in the low mass regime where we find the conventional hadronic sources. For a lepton $p_{T}$ cut of $1 \mathrm{GeV} / \mathrm{c}$ (1.h.s.) one practically cannot identify an effect of the $\rho$ collisional broadening in the dilepton spectra in the PHSD calculations. Only when applying a low $p_{T}$ cut of $0.15 \mathrm{GeV} / \mathrm{c}$ a small enhancement of the dilepton yield from 0.3 to $0.7 \mathrm{GeV}$ becomes visible (r.h.s. of Fig. 4). This low sensitivity to hadronic in-medium effects at LHC energies is due to the fact that the hadrons come out late in central $\mathrm{Pb}+\mathrm{Pb}$ collisions and are boosted to high velocities due to the high pressure in the early partonic phase. 

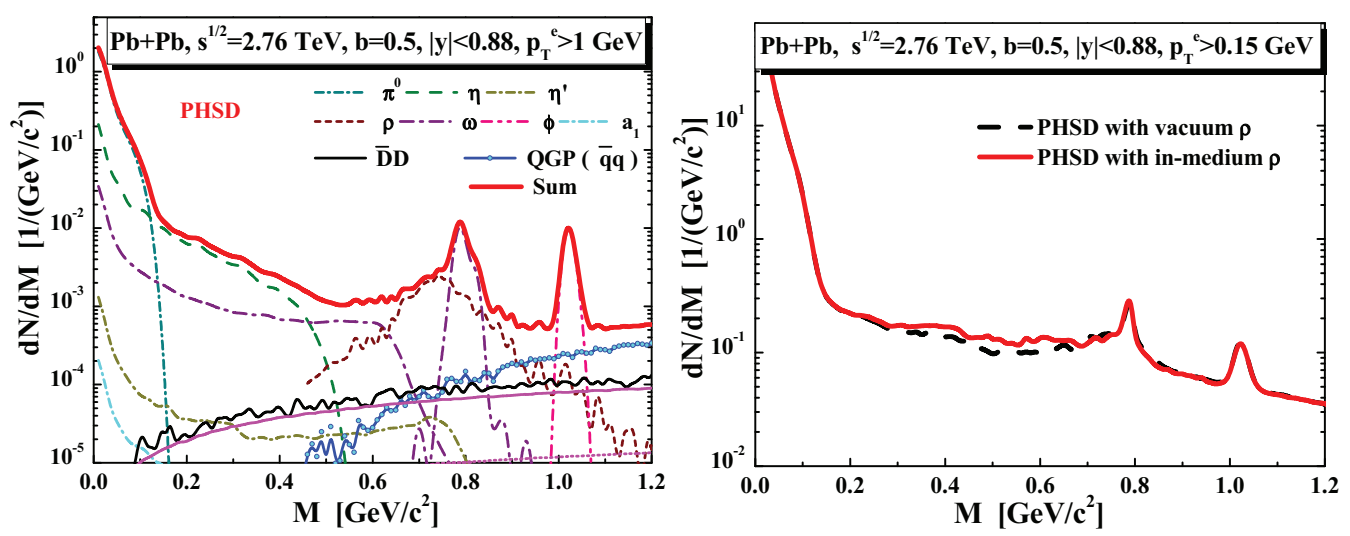

Figure 4. Midrapidity dilepton yields for $\mathrm{Pb}+\mathrm{Pb}$ at $\sqrt{s_{N N}}=2.76 \mathrm{TeV}$ (1.h.s.) for a lepton $p_{T}$ cut of $1 \mathrm{GeV} / \mathrm{c}$. The channel decomposition is explained in the legend. (r.h.s.) Same as for the 1.h.s. but for a lepton $p_{T}$ cut of $0.15 \mathrm{GeV} / \mathrm{c}$ for a 'free' $\rho$ spectral function (dashed line) and the collisional broadening scenario (solid line). The figures are taken from Ref. [34].

In the end, we mention that promising perspectives with dileptons have been suggested in Ref. [35] to measure the anisotopy coefficients $v_{n}, n=2,3$ similar to photons. The calculations with the viscous (3+1)d MUSIC hydro for central $\mathrm{Au}+\mathrm{Au}$ collisions at RHIC energies show that $v_{2}, v_{3}$ are sensitive to the dilepton sources and to the EoS and $\eta / s$ ratio. The main advantage of measuring flow coefficients $v_{n}$ with dileptons compared to photons is the fact that an extra degree of freedom $M$ might allow to disentangle the sources.

\section{Conclusions}

In conclusion, our calculations show that: i) the low mass $\left(M=0.2-0.6 \mathrm{GeV} / c^{2}\right)$ dilepton spectra show sizable changes due to hadronic in-medium effects, i.e. multiple hadronic resonance formation (at SIS energies) or a modification of the properties of vector mesons (such as collisional broadening) in the hot and dense hadronic medium (partially related to chiral symmetry restoration); these effects can be observed at all energies up to LHC (preferentially in heavy systems) but are most pronounced in the FAIR/NICA energy regime; (ii) at intermediate masses the QGP ( $q \bar{q}$ thermal radiation) dominates for $M>1.2 \mathrm{GeV} / c^{2}$, it grows with increasing energy and becomes dominant at the LHC energies. The dilepton measurements within the future experimental energy and system size scan $(p p, p A, A A)$ from low to top RHIC energies as well as new ALICE data at LHC energies will extend our knowledge on the properties of hadronic and partonic matter via its electromagnetic radiation.

The author acknowledges financial support through the 'HIC for FAIR' framework of the 'LOEWE' program and likes to thank all her coauthors for their help and valuable contributions.

\section{References}

[1] M. M. Aggarwal et al., STAR Colaboration, Phys. Rev. C71, 064902 (2005); Phys. Rev. Lett. 97, 132301 (2006); arXiv:1006.1961.

[2] W. Cassing and E. L. Bratkovskaya, Nucl. Phys. A831, 215 (2009). 
[3] W. Cassing, Nucl. Phys. A A795, 70 (2007).

[4] E. L. Bratkovskaya, W. Cassing, V. P. Konchakovski, and O. Linnyk, Nucl. Phys. A856, 162 (2011).

[5] Y. Aoki et al., JHEP 0906, 088 (2009).

[6] A. Peshier and W. Cassing, Phys. Rev. Lett. 94, 172301 (2005).

[7] W. Ehehalt and W. Cassing, Nucl. Phys. A 602, 449 (1996).

[8] W. Cassing and E. L. Bratkovskaya, Phys. Rep. 308, 65 (1999).

[9] H. U. Bengtsson and T. Sjöstrand, Comp. Phys. Commun. 46, 43 (1987).

[10] W. Cassing, Nucl. Phys. A 791, 365 (2007).

[11] W. Cassing, Eur. J. Phys. 168, 3 (2009).

[12] E.L. Bratkovskaya, Nucl. Phys. A 931, 194 (2014).

[13] E. L. Bratkovskaya and C. M. Ko, Phys. Lett. B 445, 265 (1999).

[14] G. Agakishiev et al. [HADES Collaboration], Phys. Rev. C 84, 014902 (2011).

[15] G. Agakishiev et al. [HADES Collaboration], Phys. Lett. B 663, 43 (2008).

[16] E. L. Bratkovskaya and W. Cassing, Nucl. Phys. A 807, 214 (2008).

[17] E. L. Bratkovskaya, J. Aichelin, M. Thomere, S. Vogel and M. Bleicher, Phys. Rev. C 87, 064907 (2013).

[18] G. Agakishiev et al. [HADES Collaboration], Phys. Lett. B 690, 118 (2010).

[19] T. Galatyuk et al. [HADES Collaboration], Nucl. Phys. A 931, 41 (2014).

[20] D. Adamova et al. (CERES Collaboration), Nucl. Phys. A 715, 262 (2003); Phys. Lett. B 666, 425 (2008).

[21] R. Arnaldi et al. (NA60 Collaboration), Phys. Rev. Lett. 96, 162302 (2006); Eur. Phys. J. C 61, 711 (2009).

[22] R. Rapp, Adv. High Energy Phys. 2013, 148253 (2013). R. Rapp, J. Wambach, H. van Hees, in "Relativistic Heavy-Ion Physics", edited by R. Stock, Landolt-Boernstein, Volume I/23, 4-1 (2010).

[23] G. Q. Li, C. M. Ko and G. E. Brown, Phys. Rev. Lett. 75, 4007 (1995).

[24] E. L. Bratkovskaya, W. Cassing, and O. Linnyk, Phys. Lett. B670, 428 (2009). O. Linnyk et al., Phys. Rev. C 84, 054917 (2011).

[25] J. Ruppert and T. Renk, Phys. Rev. C 71, 064903 (2005); Erratum-ibid. C 75, 059901 (2007);

K. Dusling, D. Teaney, and I. Zahed, Phys. Rev. C 75, 024908 (2007); E. Santini et al., Phys. Rev. C 84, 014901 (2011).

[26] A. Toia et al. (PHENIX Collaboration), Nucl. Phys. A 774, 743 (2006); J. Phys. G 35, 104037

(2008); A. Adare et al., Phys. Lett. B670, 313 (2009); Phys. Rev. C 81, 034911 (2010).

[27] O. Linnyk et al., Phys. Rev. C 85, 024910 (2012).

[28] R. Rapp, Adv. High Energy Phys. 2013, 148253 (2013).

[29] L. Ruan, Nucl. Phys. A 931, 185 (2014).

[30] L. Ruan et al. (STAR Collaboration), Nucl. Phys. A 855, 269 (2011).

[31] X. Dong et al. (STAR Collaboration), Nucl. Phys. A 904-905, 19c (2013); F. Geurts et al., Nucl.

Phys. A 904-905, 217c (2013).

[32] Y. Guo (for the STAR Collaboration), J. Phys. Conf. Ser. 535, 012006 (2014).

[33] M. K. Köhler (for the ALICE Collaboration), Nucl. Phys. A 931, 665 (2014).

[34] O. Linnyk et al., Phys.Rev. C 87, 014905 (2013).

[35] G. Vujanovic et al., Phys. Rev. C 89, 034904 (2014); arXiv:1408.1098. 